\title{
Photo-Thermal Induced Optical Scattering Modulation Sensor for Malaria Diagnosis
}

\author{
Daniel Maitethia Memeu1,2, Merenga Abdallah Sarroney², Ciira Maina ${ }^{3}$ \\ ${ }^{1}$ Department of Physical Sciences, Meru University of Science and Technology, Meru, Kenya \\ ${ }^{2}$ Department of Physics, Kenyatta University, Nairobi, Kenya \\ ${ }^{3}$ Dedan Kimathi University of Technology, Nyeri, Kenya \\ Email: danielmaitethia@gmail.com
}

How to cite this paper: Memeu, D.M., Sarroney, M.A. and Maina, C. (2018) Photo-Thermal Induced Optical Scattering Modulation Sensor for Malaria Diagnosis. Open Journal of Biophysics, 8, 185-193. https://doi.org/10.4236/ojbiphy.2018.84014

Received: April 27, 2018

Accepted: September 10, 2018

Published: September 13, 2018

Copyright $\odot 2018$ by authors and Scientific Research Publishing Inc. This work is licensed under the Creative Commons Attribution International License (CC BY 4.0).

http://creativecommons.org/licenses/by/4.0/

\begin{abstract}
Malaria is one of the leading killer diseases in sub-Saharan Africa. Although the disease is curable, early and accurate diagnosis is key to effective therapy. Existing malaria diagnostic techniques have low detection accuracy especially when the parasite load in the blood is low. In this paper, we report on a simple photo-thermal based technique for detection of the Plasmodium parasites' biomarker (hemozoin) in blood smear samples. The technique has demonstrated $100 \%$ Plasmodium detection sensitivity and specificity from the ten blood smear test samples used.
\end{abstract}

\section{Keywords}

Photothermal Induced Optical Scattering Modulation, Photoacoustic Probing, Hemozoin, Hemoglobin, Probe Beam, Excitation Beam

\section{Introduction}

\section{Malaria Background}

Malaria is a tropical disease that has been a leading cause of deaths in sub-Saharan Africa with children below five years being the most affected. According to World Health Organization (WHO) 2015 malaria report, the disease caused about 0.5 million deaths globally and infected approximately 200 million people during the reported period [1]. The disease is caused by a protozoan parasite of the geneus Plasmodia. There are five species of Plasmodia that infect humans namely; Plasmodium falciparum, Plasmodium vivax, Plasmodium ovale, Plasmodium malariae, and Plasmodium knowlesi. The parasites are transmitted from infected to non-infected persons through a bite by female 
anopheles mosquito. Once the parasites have been launched into the blood stream, they are transported to the liver for incubation [2]. Upon maturity the parasites are released to the blood stream where they attack red blood cells (erythrocytes), feeding on hemoglobin and depositing a by-product known as hemozoin. Hemozoin is an insoluble iron compound. The compound has been reported to possess nano-rod structure and strongly absorbs optical radiation [3]. Hemozoin therefore can serve as Plasmodium parasite biomarker in blood.

Early and accurate diagnosis of the malaria is key to effective treatment of the disease. However existing malaria diagnostic technique such as optical microscopy (which is the gold standard method) and Plasmodium parasites antigen detection assays also known as Rapid Diagnostic Techniques (RDTs) lack high detection sensitivity and specificity [2], [4]. Both methods are invasive with optical microscopy also being a time consuming process. Therefore, there is an urgent need for development of a rapid malaria diagnostic technique with capability of non-invasive operation.

Limited studies have been reported on development of non-invasive techniques for malaria diagnosis [5]-[11]. The trend from the reported literature points towards use of photothermal related techniques for probing hemozoin presence in infected erythrocytes flowing through blood vessels beneath the skin surface. Photoacoustic flow cytometry has been the most preferred option [2], [8] [9] [10] [11]. This is because photoacoustic detection techniques boast from a number of desirable features which include; high detection sensitivity due to high optical absorption contrast in absorbing molecules. Besides, the emitted acoustic signal is less immune to scattering as and therefore can form high resolution images as compared to purely optical imaging technique such as Diffuse Optical Tomography (DOT) [12], [13]. Third, optically bulk tissue can be probed in reflection mode where both the optical source and the detector can be situated on the same side of the probed sample [14] [15] [16] [17]. Other existing photo-thermal based techniques such as thermal lens effect [18] are considered not suitable for in vivo probing of hemozoin since they require use of thin transparent samples (small volumes) and forward mode detection (where the optical sources is in the opposite side of the detector with respect to the sample). These requirements render the techniques unsuitable for in vivo probing of human tissue-because of its bulkiness and the strong optical scattering involved.

Figure 1 (obtained from reference [19]) gives the schematic diagrams of photoacoustic flow cytometry and thermal lensing. PBDBEB is the diameter of the probe beam of a thermal lens setup before application of the excitation beam to the sample. PBDAEB is the diameter (divergence) of the probe beam after the application of the excitation beam. Thermal lens effect causes broadening of the probe beam and change of its intensity after the sample has been irradiated by the excitation beam. This is because when the sample absorbs optical energy from the excitation beam the energy is converted to heat and temporary confined within the illuminated region of the absorber. This causes perturbation of 


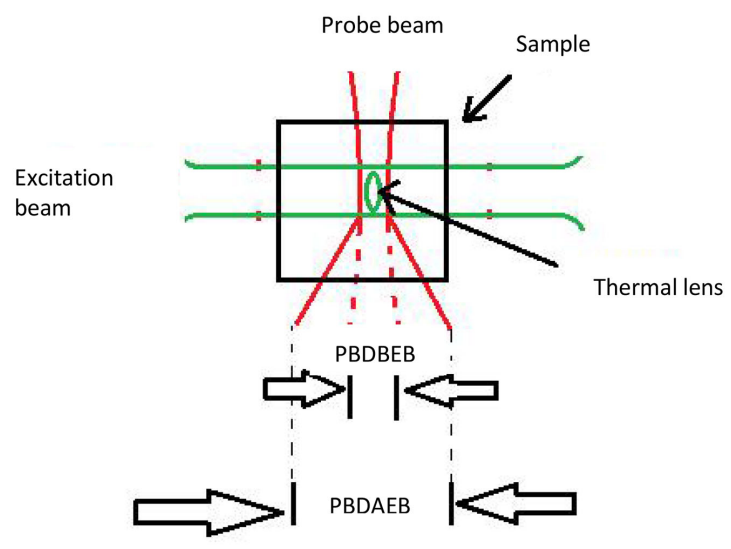

(a)

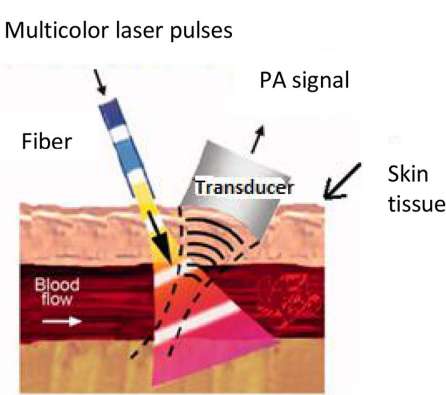

(b)

Figure 1. Configurations of thermal lensing and photoacoustic flow cytometry. (a) Perpendicular geometry of thermal lens setup [19], thermal lens effect; (b) photoacoustic flow cytometry, PA flow cytometry.

the absorber's intrinsic optical properties (specifically the refractive index) from the equilibrium (steady) state which in turn causes divergence and change of intensity of the probe beam.

The reported photoacoustic based malaria diagnostic technique on the other hand suffers from a number of limitations which has hampered its clinical adoption. One major challenge has been the problem of wide inter- and intra-variability of detected PA signals. Detected acoustic signals also suffer from low SNR due to acoustic attenuation before the signal reaches the transducer. The conversion efficiency from optical energy to acoustic energy in tissue (water) is also significantly low due to the low value of Gruneisten parameter for water which is estimated to be 0.2 [20]. This requires use of sophisticated hardware to suppress the noise and amplify the detected signal and this makes the setup complex, bulky and expensive.

In this paper, we report development of a novel technique termed as Photothermal Induced Optical Scattering Modulation (PTIOSM) for detection of hemozoin in malaria infected blood. The technique is premised on the fact that photon absorption by chromophores is followed by a relaxation session where the absorber's molecules move from excited state to ground state. If the relaxation process is non-radiative, the absorbed optical energy is released in form of heat. The emitted heat energy takes some time before it diffuses away from where it is deposited (Thermal conferment time). During this phase, the intrinsic optical properties such as the absorber's refractive index and its optical absorption coefficient are temporarily modified.

In the case of PTIOSM technique, modulation in the probe beam signal is detected in the reflection (backward) mode instead of transmission (forward) mode as is the case for thermal lens deflection. Modulation intensity with respect to optical excitation wavelength is expected to carry information on the composition as well as the concentration on chromophores in the probed sample. 


\section{Materials and Methods}

\subsection{PTIOSM Set-Up}

A common anode, $3 \mathrm{~W}$ - $9 \mathrm{~W}$ RGB Light Emitting Diode (LED) (product ID 2524, from Adafruit Industries, USA [21]) was used as the optical source for providing both the Probe Beam (PB) and the Excitation Beam (EB). The PB was produced by forward biasing the blue LED segment with a constant $350 \mathrm{~mA}$ current to generate blue light whose peak wavelength was $465 \mathrm{~nm}$. The EB beam was produced by driving the red and green LED segments with $1 \mathrm{MHz}$ square pulses having $40 \mathrm{nS}$ pulse width. The input signal for driving the LED segments was from a DDS function generator (KKmoon $40 \mathrm{MHz}$ Function Signal Generator Dual-channel DDS Arbitrary Waveform Pulse Signal Generator $1 \mathrm{~Hz}-100$ $\mathrm{MHz}$ Frequency Meter) that was coupled to a custom made RGB LED driver circuit. Figure 2 gives the block diagram of the setup. Both the red and green LED segments were overdriven with $2 \mathrm{~A}$ forward current during the $\mathrm{ON}$ period of the driving waveform in order to produce sufficient optical intensity for generation of PTIOSM signal.

Light from the RGB LED (PB and EB) irradiated samples (stained blood smear slides) and the transmitted light passed through an optical filter (Omega Optical, Inc. $450 \mathrm{~nm}$ SP (450 SP) RapidEdge $25 \mathrm{~mm}$ Optical Shortpass Filter) that blocked the EB (the red and green pulsed light) but transmitted the probe beam (the blue light) after interacting with the photo-thermally excited sample. A photodiode and trans-impedance amplifier module (OPT101) was used to detect light from the filter and the generated photodiode current was converted to a voltage signal and then pre-amplified using an inbuilt trans-impedance amplifier in the module. To boost the bandwidth of the detected signal, a $100 \mathrm{ohms}$ resistor was externally connected to the trans-impedance module (between pin 2 and 5 while pin 4 was left unconnected) to provide the negative feedback instead of using the inbuilt $1 \mathrm{M} \Omega$ resistor that offers a limited bandwidth of $12 \mathrm{KHz}$ [22]. The output of the trans-impedance amplifier was coupled to a digital oscilloscope (OWON SDS1052, 2 channel, $500 \mathrm{MS} / \mathrm{s}, 10 \mathrm{~KB}$ recording length) for digitization. The digitized signal was then loaded to a computer for signal processing. Matlab R2015b software was used for processing the acquired PTIOSM signals. Figure 3 gives the algorithmic steps used for signal processing.

\subsection{Signal Pre-Processing}

The aim of this stage was to reduce or possibly eliminate the noise riding in the acquired PTIOSM signal. There were two possible main sources of noise in the acquired signals; the radiative pick-up noise from adjacent switching equipment such as the function generator, and the background (stray) light detected by the photodiode. Noise correction was performed by coherently subtracting the PTIOSM signal due to a blank glass slide (also termed as the reference sample) from a PTIOSM signal obtained from a stained blood smear sample. The resultant differential signal was free of existing common mode noise. 

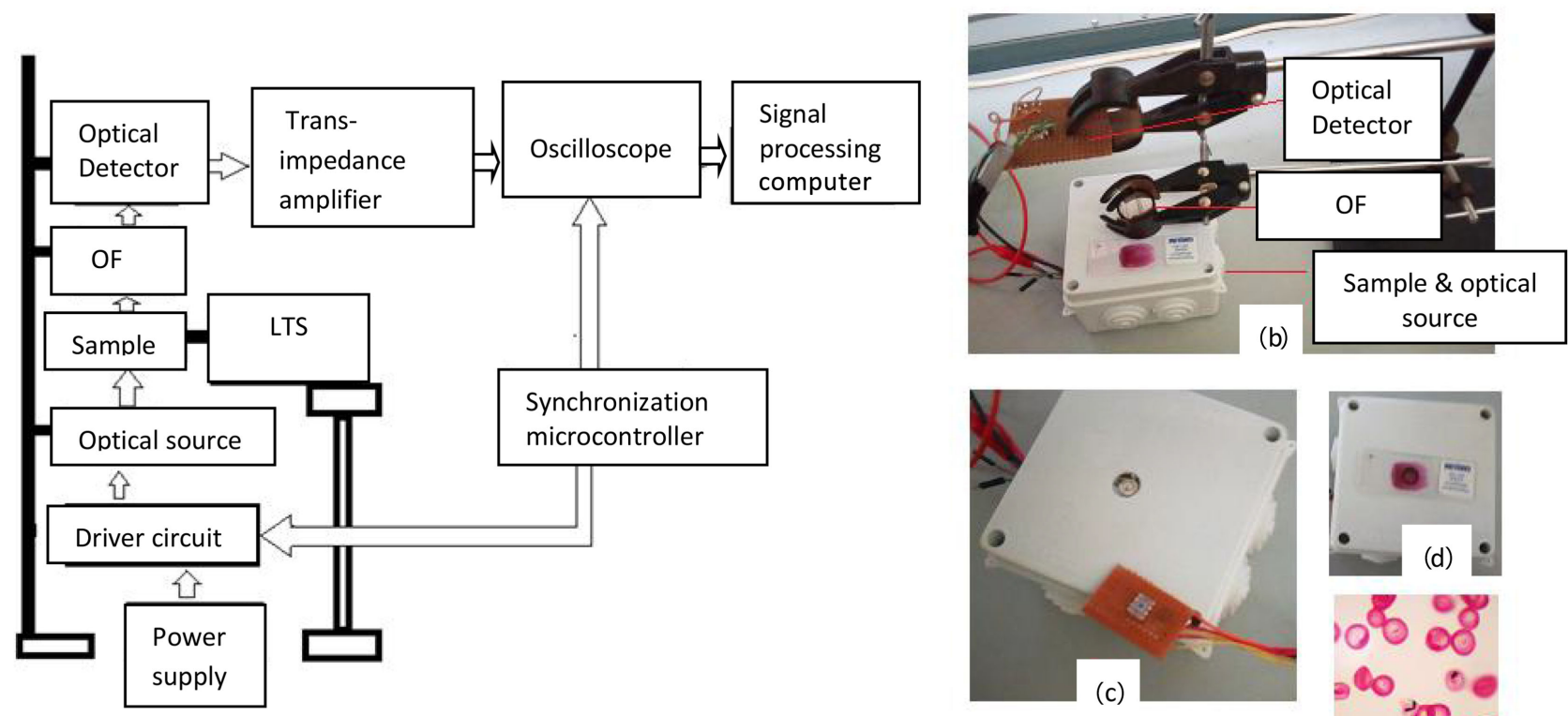

(a)

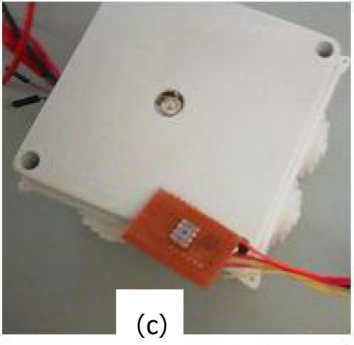

(c)

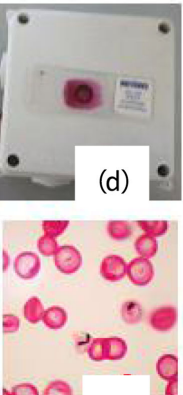

(e)

Figure 2. Setup diagram of the PTIOSM probe for malaria diagnosis, (a) setup block diagram: OF (optical Filter), LTS (Linear translational stage); (b) photo of the setup showing optical source, sample, optical filter and photodiode circuit; (c) Optical source and detector; (d) blood smear sample; (e) microscopic view of the sample.

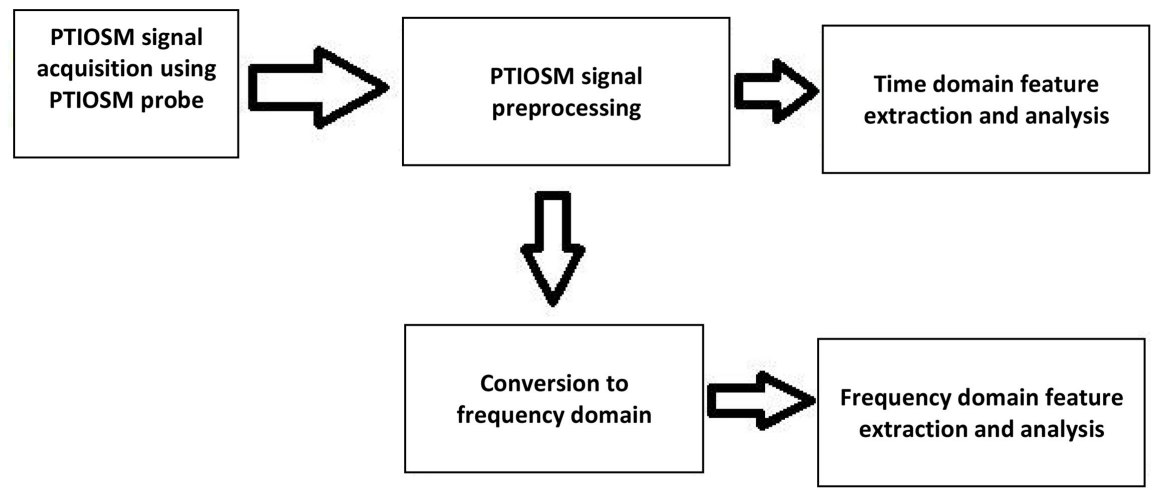

Figure 3. Algorithmic steps for PTIOSM signal processing.

\subsection{Frequency Domain Feature Extraction}

Fast Fourier Transform (FFT) algorithm implemented in Matlab was used to convert the acquired time domain signals to frequency domain. The amplitude and phase spectrums of the frequency domain signals were plotted in Matlab and visually analyzed with a view of identifying the frequency bands which best differentiated infected samples from non-infected samples based on their signal intensities at different frequency bands.

\subsection{Sample Description}

Two sets of Geimsa stained blood smear samples were used to test the developed PTIOSM probe. One was Plasmodium falciparum infected blood smears samples (acquired from Carolina biological company [23]) and the other was Plasmo- 
dium non-infected blood smear samples locally prepared. In each of the two sets, PTIOSM signals from five slides were acquired, processed and analyzed as described above.

\section{Results}

\section{Frequency Domain PTIOSM Signals}

After conversion from time domain to frequency domain and noise filtering of PTIOSM signals, scatter plots for the amplitude spectra belonging to the five Plasmodium positive and five Plasmodium negative samples were made (Figure 4). From the scatter plots the following observations were made: At some frequency bands of the PTIOSM signal, the Plasmodium positive PTIOSM signals had more spectral energy compared to Plasmodium negative PTIOSM signals and therefore it was possible to find a threshold value that differentiated infected samples from the non-infected ones. In the case of the pulsed red light excitation (Figure 4(a)), 100\% classification accuracy of Plasmodium positive samples from Plasmodium negative samples was possible at frequency band between 1.7 $\mathrm{MHz}$ and $1.8 \mathrm{MHz}$ because the signal amplitudes of all infected samples were greater than those of Plasmodium negative samples. This means that at this frequency band, the Plasmodium parasite detection sensitivity and specificity was $100 \%$ (there were no false positives or false negatives). For the PTIOSM signals due to pulsed green light excitation (Figure 4(b)), the best classification accuracy was observed at frequency band between $1.5 \mathrm{MHz}$ and $1.6 \mathrm{MHz}$. At this frequency band, the signal intensities from all the five Plasmodium positive samples were greater than the Plasmodium negative signals except for one Plasmodium negative sample signal whose amplitude was comparable to those of positive samples. This represents a classification specificity of $100 \%$ and sensitivity of $83.3 \%$.

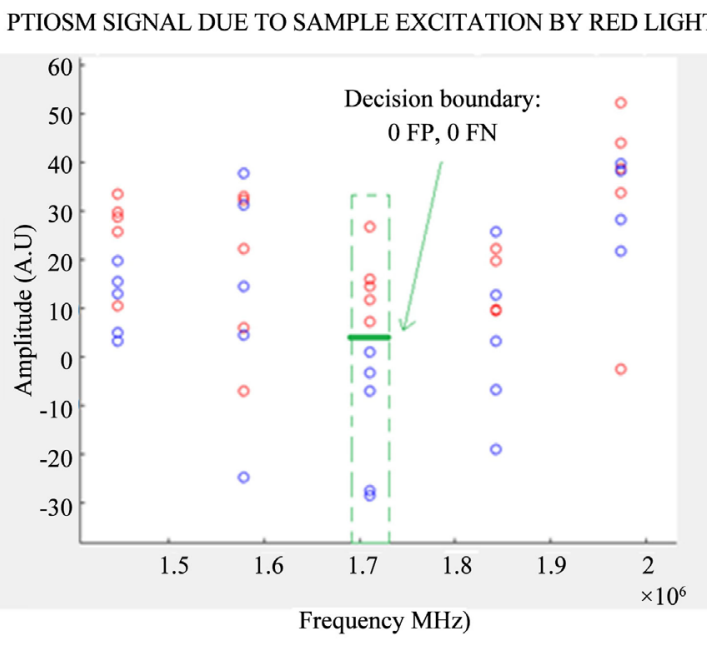

(a)
PTIOSM SIGNAL DUE TO SAMPLE EXCITATION BY GREEN LIGHT

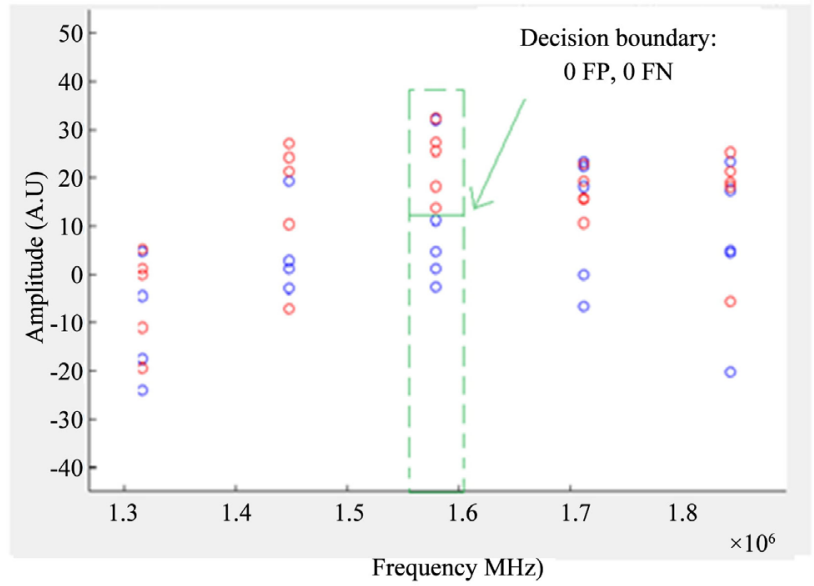

(b)

Figure 4. Frequency spectra of PTIOSM signal for malaria infected (red circles) and non-infected samples (blue circles). (a) PTIOSM signal due to red light excitation; (b) PTIOSM signal to blue light modulation. FP is false positive samples and $\mathrm{FN}$ is false negative samples. 


\section{Discussion}

PTIOSM technique ability to correctly classify malaria infected blood smear samples from the non-infected samples can be attributed to two factors; change of hemozoin's refractive index during optical excitation hence causing a significant modulation of the PTIOSM signal at frequency band corresponding to its size. Another possible explanation could be that the excitation beam causes generation of photoacoustic waves, which in turn induces vibration of optical scatterer in the medium leading to modulation of diffuse reflected light from the sample. Variations of signal intensity from samples having the same infection status (either infected or non-infected) can be attributed to two possible factors: the concentration of responsible chromophores (in this case hemozoin and hemoglobin) and the optical excitation intensity.

From the reported results, the developed PTIOSM probe has demonstrated excellent capability of malaria diagnosis in blood smear samples. The simple instrumentation involved and its real time operation makes it a suitable candidate for mass screening of the disease in malaria endemic regions. However, further testing using more samples prepared under different conditions (both thin and thick blood smears, stained and non-stained) are required to conclusively determine the techniques sensitivity and specificity. Besides, further experimentation is required to investigate the capability of the probe in quantifying the parasite load (parasitemia) in the samples and the limit of detection of the technique.

\section{Conclusion}

A novel, simple photo-thermal based technique (PTIOSM) for detection of Plasmodium parasites in infected blood smear samples has been described. A RGB LED was used as the optical source to supply both the PB and the EB. Diffusely reflected PB light from the sample was detected by a photodiode, amplified and the signal preprocessed for noise cancellation. Fourier transform was used to convert the signals from time domain to frequency domain and then some specific frequency bands were used to differentiate Plasmodium infected samples from non-infected samples based on an empirically determined intensity threshold. The technique yielded $100 \%$ detection sensitivity and specificity using red LED light as the excitation beam.

\section{Conflicts of Interest}

The authors declare no conflicts of interest regarding the publication of this paper.

\section{References}

[1] World Health Organization (2015) World Malaria Report 2015.

[2] https://www.cdc.gov/malaria/about/biology/index.html

[3] Samson, E.B., Goldschmidt, B.S., Whiteside, P.J., Sudduth, A.S., Custer, J.R., Beerntsen, B. and Viator, J.A. (2012) Photoacoustic Spectroscopy of $\beta$-Hematin. 
Journal of Optics, 14, 065302. https://doi.org/10.1088/2040-8978/14/6/065302

[4] Makler, M., Palmer, C. and Ager, A. (1998) A Review of Practical Techniques for the Diagnosis of Malaria. Annals of Tropical Medicine and Parasitology, 92, 419-433.

[5] Scholl, P.F., Kongkasuriyachai, D., Demirev, P.A., Feldman, A.B., Lin, J.S., Sullivan, J.D. and Kumar, N. (2004) Rapid Detection of Malaria Infection in Vivo by Laser Desorption Mass Spectrometry. The American Journal of Tropical Medicine and Hygiene, 71, 546-51.

[6] Bélisle, J.M., Costantino, S., Leimanis, M.L., Bellemare, M.J., Bohle, D.S., Georges, E. and Wiseman, P.W. (2008) Sensitive Detection of Malaria Infection by Third Harmonic Generation Imaging. Biophysical Journal, 94, L26-L28.

https://doi.org/10.1529/biophysj.107.125443

[7] Burnett, J.L., Carns, J.L. and Richards-Kortum, R. (2017) Towards a Needle-Free Diagnosis of Malaria: In Vivo Identification and Classification of Red and White Blood Cells Containing Haemozoin. Malaria Journal, 16, 447. https://doi.org/10.1186/s12936-017-2096-1

[8] Saha, R.K., Karmakar, S. and Roy, M. (2012) Computational Investigation on the Photoacoustics of Malaria Infected Red Blood Cells. PLoS ONE, 7, e51774.

https://doi.org/10.1371/journal.pone.0051774

[9] Lukianova-Hleb, E.Y., Campbell, K.M., Constantinou, P.E., Braam, J., Olson, J.S., Ware, R.E., Sullivan, D.J. and Lapotko, D.O. (2014) Hemozoin-Generated Vapor Nanobubbles for Transdermal Reagent- and Needle-Free Detection of Malaria. Proceedings of the National Academy of Sciences, 111, 900-905. https://doi.org/10.1073/pnas.1316253111

[10] Cai, C., Carey, K.A., Nedosekin, D.A., Menyaev, Y.A., Sarimollaoglu, M., Galanzha, E.I., Stumhofer, J.S. and Zharov, V.P. (2016) In Vivo Photoacoustic Flow Cytometry for Early Malaria Diagnosis. Cytometry Part A, 89, 531-542. https://doi.org/10.1002/cyto.a.22854

[11] Lukianova-Hleb, E., Bezek, S., Szigeti, R., Khodarev, A., Kelley, T., Hurrell, A., Berba, M., Kumar, N., D’Alessandro, U. and Lapotko, D. (2015) Transdermal Diagnosis of Malaria Using Vapor Nanobubbles. Emerging Infectious Diseases, 21, 1122. https://doi.org/10.3201/eid2107.150089

[12] Beard, P. (2011) Biomedical Photoacoustic Imaging. Interface Focus, rsfs20110028.

[13] Xia, J., Yao, J. and Wang, L.V. (2014) Photoacoustic Tomography: Principles and Advances. Progress in Electromagnetics Research, 147, 1. https://doi.org/10.2528/PIER14032303

[14] Deán-Ben, X.L. and Razansky, D. (2013) Functional Optoacoustic Human Angiography with Handheld Video Rate Three Dimensional Scanner. Photoacoustics, 1, 68-73. https://doi.org/10.1016/j.pacs.2013.10.002

[15] Zhou, Y., Li, G., Zhu, L., Li, C., Cornelius, L.A. and Wang, L.V. (2015) Handheld Photoacoustic Probe to Detect Both Melanoma Depth and Volume at High Speed in Vivo. Journal of Biophotonics, 8, 961-967. https://doi.org/10.1002/jbio.201400143

[16] Kuniyil Ajith Singh, M., Steenbergen, W. and Manohar, S. (2016) Handheld Probe-Based Dual Mode Ultrasound/Photoacoustics for Biomedical Imaging. In: Olivo, M. and Dinish, U.S., Eds., Frontiers in Biophotonics for Translational Medicine, Vol. 3, Springer, Singapore, 209-247.

[17] Daoudi, K., Van Den Berg, P.J., Rabot, O., Kohl, A., Tisserand, S., Brands, P. and Steenbergen, W. (2014) Handheld Probe Integrating Laser Diode and Ultrasound 
Transducer Array for Ultrasound/Photoacoustic Dual Modality Imaging. Optics Express, 22, 26365-26374.

[18] Liu, M. and Franko, M. (2014) Progress in Thermal Lens Spectrometry and Its Applications in Microscale Analytical Devices. Critical Reviews in Analytical Chemistry, 44, 328-353.

[19] Canto, F., Couston, L., Magnaldo, A., Broquin, J.-E. and Signoret, P. (2008) Thermal Lens Spectroscopy as a "New" Analytical Tool for Actinide Determination in Nuclear Reprocessing Processes.

[20] Wang, L.V. and Wu, H. (2007) Biomedical Optics: Principles and Imaging. John Wiley \& Sons, Hoboken.

[21] https://www.adafruit.com/product/2524

[22] http://www.ti.com/lit/ds/symlink/opt101.pdf

[23] https://www.carolina.com/protists-microscope-slides/Plasmodium-falciparum-slide -smear/297190.pr 